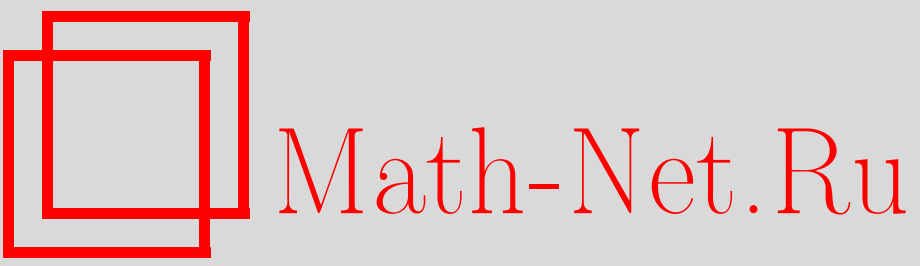

А. А. Арсеньев, Математическая модель резонансов и туннелирования в системе со связанным состоянием, ТМФ, 2003, том 136, номер 3, 507-516

DOI: https://doi.org/10.4213/tmf1915

Использование Общероссийского математического портала Math-Net.Ru подразумевает, что вы прочитали и согласны с пользовательским соглашением

http://www . mathnet.ru/rus/agreement

Параметры загрузки:

IP : 52.6 .47 .48

26 апреля 2023 г., 05:22:12 
ТЕОРЕТИЧЕСКАЯ

И МАТЕМАТИЧЕСКАЯ

ФИЗИКА

Том 136, № 3

сентябрь, 2003

(C) 2003 г.

А. А. Арсеньев*

\section{МАТЕМАТИЧЕСКАЯ МОДЕЛЬ РЕЗОНАНСОВ И ТУННЕЛИРОВАНИЯ В СИСТЕМЕ СО СВЯЗАННЫМ СОСТОЯНИЕМ}

Изучается асимптотика вычета в полюсе аналитического продолжения матрицы рассеяния при стремлении мнимой части полюса к нулю для того случая, когда фазовое пространство квантово-механической системы есть прямая сумма двух пространств, оператор невозмушенной эволюции приводит каждое из этих пространств и в одном из них имеет дискретный спектр, а в другом - непрерывный. Оператор возмущения смешивает подпространства и порождает резонанс. Доказывается, что в такой системе при выполнении определенных условий симметрии амплитуда рассеяния будет резко меняться в окрестности действительной части полюса матрицы рассеяния, и в системе будет наблюдаться туннелирование или резонанс амплитуды рассеяния.

Ключевые слова: рассеяние, резонанс, туннелирование.

\section{1. ВВЕДЕНИЕ}

Наша работа посвящена математической теории рассеяния для “систем с невылетанием" (potential scattering with permanently confined channels), cм. [1]-[3]. В середине семидесятых годов подобные системы изучались в связи с моделированием невылетания кварков, теперь они используются в квантовой теории твердого тела. "Системы с невылетанием" являются частным случаем моделей Паули-Фирца, и принцип их построения изложен в работе [4]. В последнее время такие модели стали изучать в рамках формализма "систем $2 \times 2$ ". Поясним полученные в настоящей работе результаты на примере. Рассмотрим квантово-механическую систему, фазовое пространство которой есть $\mathbb{C}^{1} \oplus L^{2}\left(\mathbb{R}^{1}, d x\right)$, и гамильтониан невозмушенной эволюции имеет вид

$$
H=\left(\begin{array}{cc}
\lambda_{\infty} & 0 \\
0 & L_{\infty}
\end{array}\right)
$$

где $L_{\infty}=-\partial_{x x}^{2}+\kappa v(x), v(x) \geqslant 0, v(-x)=v(x), \lambda_{\infty}>0, \operatorname{supp} v(x) \subset(-R, R), R<\infty$. Пусть оператор возмушения $\epsilon H_{\text {int }}$ смешивает вакуумное $\mathbb{C}^{1} \oplus 0$ и одночастичное $0 \oplus$ $L^{2}\left(\mathbb{R}^{1}, d x\right)$ подпространства (по другой терминологии пространство $\mathbb{C}^{1} \oplus 0$ называют связанным каналом (vacuum sector), а пространство $0 \oplus L^{2}\left(\mathbb{R}^{1}, d x\right)$ - радиационным

\footnotetext{
* Московский государственный университет, Москва, Россия
} 
каналом (radiation sector)), коммутирует с операцией отражения: $I \psi(x)=\psi(-x)$, и удовлетворяет условию $\operatorname{supp} v(x) \cap \operatorname{supp} H_{\text {int }}=\varnothing$. Из сформулированной в разделе 4 теоремы следует, что в такой системе при $\kappa=0,0<|\epsilon| \ll 1$ одночастичное возбуждение с энергией $\lambda \neq \lambda_{\infty}$ будет проходить из $-\infty$ в $+\infty$ почти без отражения, а одночастичное возбуждение с энергией $\lambda \approx \lambda_{\infty}$ будет почти полностью отражаться. При $\kappa \max _{x} v(x) \gg \lambda_{\infty}, \quad 0<|\epsilon| \ll 1$ будет наблюдаться туннелирование: одночастичное возбуждение с энергией $\lambda \approx \lambda_{\infty}$ будет проходить из $-\infty$ в $+\infty$ почти без отражения, а одночастичное возбуждение с энергией $\lambda \neq \lambda_{\infty}, \lambda<\kappa \max _{x} v(x)$, будет отражаться от потенциального барьера. Ясно, что условие симметрии системы относительно отражения для этого существенно. В работе предложен способ учета влияния подобной симметрии на поведение амплитуды рассеяния вблизи действительной части полюса матрицы рассеяния.

Традиционно задача туннелирования изучается квазиклассическими методами. Наш подход никак с ними не связан.

С математической точки зрения наша модель описывает рассеяние в гильбертовом пространстве

$$
\mathcal{H}=\mathcal{H}_{1} \oplus \mathcal{H}_{2}, \quad \mathcal{H}_{1} \perp \mathcal{H}_{2}
$$

Предполагается, что разбиение (1) приводит оператор эволюции невозмушенной системы $H_{0}$, сужение оператора $H_{0}$ на $\mathcal{H}_{2}$ имеет абсолютно непрерывный спектр, спектр сужения $H_{0}$ на $\mathcal{H}_{1}$ дискретен и лежит в непрерьвном спектре сужения $H_{0}$ на $\mathcal{H}_{2}$, а оператор возмушения $V$ смешивает пространства $\mathcal{H}_{1}$ и $\mathcal{H}_{2}$ и порождает резонанс.

Изучение подобных моделей было начато Фридрихсом [5] и Лифшшицем (современное изложение его результатов есть в [6]). Эти модели интенсивно исследуются и сейчас (см. [1]-[4], [7]-[10]). Обычно изучается спектральная функция возмушенного оператора или его функция Грина. Мы считаем эту задачу уже решенной (мы налагаем условия, при которых она тривиальна) и изучаем асимптотику вычета в полюсе матрицы рассеяния при стремлении мнимой части полюса к нулю. Вычет в полюсе матрицы рассеяния рассматривался во многих работах ([6], [7], [11], [12]); мы приводим тот вариант известных формул, из которого удобно извлечь нужную нам информацию. Основной результат нашей работы изложен в теореме (см. раздел 4).

Применяемые обозначения стандартны, поясним лиш некоторые из них. Скалярное произведение в гильбертовом пространстве мы считаем линейным по второму аргументу. Символ $\|\quad \mid B\|$ означает норму в банаховом пространстве $B$, если указание на пространство необходимо. Символ $L(A \rightarrow B)$ означает банахово пространство линейных непрерывных операторов из банахова пространства $A$ в банахово пространство $B$. Если гильбертово пространство $\mathcal{H}$ представлено как ортогональная сумма $(1)$, то $\psi_{\alpha}$ - это компонента вектора $\psi$ в пространстве $\mathcal{H}_{\alpha},\langle,\rangle_{\alpha}$ - сужение скалярного произведения на $\mathcal{H}_{\alpha}$,

$$
(A \psi)_{\alpha}=\sum_{\beta} A_{\alpha \beta} \psi_{\beta}, \quad A_{\alpha \beta} \in L\left(\mathcal{H}_{\beta} \rightarrow \mathcal{H}_{\alpha}\right)
$$

$\alpha$ - номер строки, $\beta$ - номер столбца. 


\section{2. ОПИСАНИЕ МОДЕЛИ}

Мы собираемся изучать матрицу рассеяния для операторов эволюции $H_{0}, H_{0}+V$. Обычно оператор $H_{0}$ не ограничен, а оператор $V$ - не ядерный. Если можно так подобрать удовлетворяющую условиям принципа инвариантности волновых операторов функцию $F$, что оператор $F\left(H_{0}\right)$ будет ограничен, а оператор $F\left(H_{0}+V\right)-F\left(H_{0}\right)-$ ядерный, то изучение локального поведения матришы рассеяния для пары $H_{0}, H_{0}+V$ можно свести к более простой задаче изучения матрицы рассеяния для пары $F\left(H_{0}\right)$, $F\left(H_{0}+V\right)$. Мы будем считать, что такое сведение уже сделано.

Пусть $L$ - самосопряженный оператор в $\mathcal{H}_{2}$. Мы предположим, что спектр оператора $L$ абсолютно непрерывен. Наши дальнейшие предположения об операторе $L$ описывают известную конструкцию [13] спектрального разложения оператора с абсолютно непрерывным спектром.

Пусть $\Omega$ - компактное топологическое пространство, $d \omega$ - неотрицательная борелевская мера на $\Omega$, скалярное произведение в $L^{2}(\Omega, d \omega)$ мы обозначим символом $\langle\cdot, \cdot\rangle_{\Omega}$. Пусть $h$ - гильбертово пространство интегрируемых с квадратом функций на $(a ; b)$ со значениями в $L^{2}(\Omega, d \omega)$ :

$$
\langle f, g\rangle_{h}=\int_{a}^{b}\langle f(\lambda), g(\lambda)\rangle_{\Omega} d \lambda .
$$

Пусть сушествует такой унитарный оператор $U \in L\left(\mathcal{H}_{2} \rightarrow h\right)$, что оператор $L$ унитарно-эквивалентен оператору умножения на $\lambda$ :

$$
\langle f, L g\rangle_{2}=\int_{a}^{b} \lambda\langle U f(\lambda), U g(\lambda)\rangle_{\Omega} d \lambda .
$$

Мы предположим, что обратный оператор $U^{-1} \in L\left(h \rightarrow \mathcal{H}_{2}\right)$ сушествует и в пространстве $h$ действует как интегральный оператор с ядром $U^{-1}(\lambda, \omega)$.

Таким образом, $\Omega$ - это пространство, которое параметризует собственные функшии непрерывного спектра оператора $L, U f(\lambda, \omega)$ - коэффициент Фурье элемента $f \in \mathcal{H}_{2}$, $U^{-1}(\lambda, \omega)$ - биортогональная система собственных функций оператора $L$.

Пусть гильбертовы пространства $\mathcal{H}_{2}^{ \pm}$таковы, что

$$
\mathcal{H}_{2}^{-} \subset \mathcal{H}_{2} \subset \mathcal{H}_{2}^{+}
$$

и выполнены следующие условия.

1. Пространство $\mathcal{H}_{2}^{-}$плотно в $\mathcal{H}_{2}$, пространство $\mathcal{H}_{2}$ плотно в $\mathcal{H}_{2}^{+}$и форма $\langle\cdot, \cdot\rangle_{2}$ приводит $\mathcal{H}_{2}^{-}$и $\mathcal{H}_{2}^{+}$в двойственность.

2. Функция

$$
\left(\lambda_{\infty}-\delta, \lambda_{\infty}+\delta\right) \times \Omega \ni(\lambda \times \omega) \rightarrow U^{-1}(\lambda, \omega) \in \mathcal{H}_{2}^{+}
$$

аналитична по $\lambda$ при фиксированном $\omega$ в не зависящей от $\omega$ комплексной окрестности интервала $\left(\lambda_{\infty}-\delta, \lambda_{\infty}+\delta\right) \subset(a ; b)$.

В дальнейшем мы будем считать, что эта окрестность есть круг $B_{\delta}=\{\lambda$ : $\left.\left|\lambda_{\infty}-\lambda\right|<\delta\right\}$. Выбор точки $\lambda_{\infty}$ пояснен ниже равенством (6).

3. Функция

$$
B_{\delta} \ni \lambda \rightarrow R(\lambda, L)=(\lambda-L)^{-1} \in L\left(\mathcal{H}_{2}^{-} \rightarrow \mathcal{H}_{2}^{+}\right)
$$


имеет аналитическое продолжение из верхней полуплоскости в нижнюю (мы обозначим его $R^{+}(\lambda, L)$ ) и из нижней полуплоскости в верхнюю (это продолжение мы обозначим $\left.R^{-}(\lambda, L)\right)$.

Заметим, что тройка (2) может отличаться от стандартного оснашения: мы не требуем ядерности вложения $\mathcal{H}_{2}^{-} \rightarrow \mathcal{H}_{2}^{+}$.

Положим

$$
\mathcal{H}^{ \pm}=\mathcal{H}_{1} \oplus \mathcal{H}_{2}^{ \pm}
$$

Мы называем самосопряженный ядерный оператор $V \in L(\mathcal{H} \rightarrow \mathcal{H})$ допустимьлм, если он продолжается по непрерьвности на $\mathcal{H}^{+}, V \mathcal{H}^{+} \subset \mathcal{H}^{-}$, и оператор $V$ вполне непрерывен как оператор из $L\left(\mathcal{H}^{+} \rightarrow \mathcal{H}^{-}\right)$.

В дальнейшем мы предположим, что

$$
\operatorname{dim} \mathcal{H}_{1}=m<\infty
$$

В этом случае матричные элементы оператора $V$ имеют вид

$$
\begin{aligned}
& V_{21} f=\sum_{1 \leqslant j \leqslant m}\left\langle e_{j}, f\right\rangle_{1} \eta_{j}, \quad \eta_{j} \in \mathcal{H}_{2}^{-}, \\
& V_{12} f=\sum_{1 \leqslant j \leqslant m}\left\langle\eta_{j}, f\right\rangle_{2} e_{j}, \quad\left\langle e_{i}, e_{j}\right\rangle_{1}=\delta_{i j} .
\end{aligned}
$$

4. Пусть $V_{\infty}$ - допустимый оператор и $V_{n}$ - последовательность допустимых операторов, которая удовлетворяет следующим условиям:

a) $V_{n, 11} \rightarrow V_{\infty, 11}, n \rightarrow \infty$;

б) $\left\|\left(V_{n, 22}-V_{\infty, 22}\right) \mid L\left(\mathcal{H}_{2}^{+} \rightarrow \mathcal{H}_{2}^{-}\right)\right\| \rightarrow 0, n \rightarrow \infty$;

в) если $\eta_{n, j}$ - функции, которые задают операторы $V_{n, 21}, V_{n, 12}$ по формулам (3), то

$$
\left\|\eta_{n, j} \mid \mathcal{H}_{2}^{-}\right\| \rightarrow 0, \quad n \rightarrow \infty
$$

5. При $n<\infty$ выполнено неравенство

$$
\left\|\sum_{1 \leqslant j \leqslant m}\left\langle e_{j}, \psi_{\infty}\right\rangle U \eta_{n, j}\left(\lambda_{\infty}, \omega\right) \mid L^{2}(\Omega, d \omega)\right\|>0 .
$$

6. Оператор $V_{\infty, 11}$ имеет простое собственное значение

$$
V_{\infty, 11}\left(\psi_{\infty}\right)_{1}=\lambda_{\infty}\left(\psi_{\infty}\right)_{1}, \quad \lambda_{\infty} \neq 0, \quad \lambda_{\infty} \in(a ; b) .
$$

В дальнейшем мы будем отмечать индексом $n$ величины, которые соответствуют оператору $V_{n}$.

Пусть

$$
\begin{array}{ll}
H_{0}=\left(\begin{array}{cc}
0 & 0 \\
0 & L
\end{array}\right), & R^{ \pm}\left(\lambda, H_{0}\right)=\left(\begin{array}{cc}
\lambda^{-1} & 0 \\
0 & R^{ \pm}(\lambda, L)
\end{array}\right), \\
H_{n}=H_{0}+V_{n}, & \Gamma_{n}^{ \pm}(\lambda)=R^{ \pm}\left(\lambda, H_{0}\right) V_{n} .
\end{array}
$$


Если $\left(\psi_{\infty}\right)_{1}$ есть решение уравнения $(6)$, то вектор

$$
\psi_{\infty}=\left(\psi_{\infty}\right)_{1} \oplus 0
$$

удовлетворяет уравнениям

$$
\begin{aligned}
\psi_{\infty} & =\Gamma_{\infty}^{ \pm}\left(\lambda_{\infty}\right) \psi_{\infty}, & & \psi_{\infty} \in \mathcal{H}^{+} \\
H_{\infty} \psi_{\infty} & =\lambda_{\infty} \psi_{\infty}, & & \psi_{\infty} \in \mathcal{H}^{+}
\end{aligned}
$$

Далее мы сделаем следующие предположения.

7. Уравнение (8) имеет единственное линейно независимое нетривиальное решение.

Если оператор $L$ может быть продолжен по замыканию на $\mathcal{H}_{2}^{+}$(см. [11]), то это эквивалентно предположению о том, что уравнение

$$
\left(L+V_{\infty}\right)\left(\psi_{\infty}\right)_{2}=\lambda_{\infty}\left(\psi_{\infty}\right)_{2}, \quad\left(\psi_{\infty}\right)_{2} \in \mathcal{H}_{2}^{+}
$$

не имеет нетривиальных решений, представимых в виде

$$
\left(\psi_{\infty}\right)_{2}=R^{ \pm}\left(\lambda_{\infty}, L\right) f, \quad f \in \mathcal{H}_{2}^{-} .
$$

8. Существует гомоморфизм $l: \mathcal{H}^{+} \rightarrow \mathcal{H}^{+}$, удовлетворяющий следующему условию: в некоторой комплексной окрестности точки $\mu=1$ при $\lambda \in B_{\delta}, n \geqslant n_{0}$ в пространстве $\mathcal{H}^{+}$выполнены равенства

$$
\begin{gathered}
l(\mu \psi)=\bar{\mu} l(\psi), \quad l\left(\operatorname{Ker}\left(\bar{\mu}-\Gamma_{n}^{-}(\bar{\lambda})\right)\right)=\operatorname{Ker}\left(\mu-\Gamma_{n}^{+}(\lambda)\right), \\
l\left(\operatorname{Ker}\left(\mu-\Gamma_{n}^{+}(\lambda)\right)\right)=\operatorname{Ker}\left(\bar{\mu}-\Gamma_{n}^{-}(\bar{\lambda})\right) .
\end{gathered}
$$

9. Существует такое непрерывное отображение $p: \Omega \rightarrow \Omega$, что для всех $\omega \in \Omega, \lambda \in$ $B_{\delta}, n \geqslant n_{0}, \psi \in \operatorname{Ker}\left(\mu-\Gamma_{n}^{ \pm}(\lambda)\right)$

$$
\left|\left\langle V_{n} l^{-1}(\psi), U^{-1}(\lambda, p(\omega))\right\rangle_{2}\right|=\left|U\left(V_{n} \psi\right)_{2}(\lambda, \omega)\right| .
$$

\section{3. СВОЙСТВА МОДЕЛИ}

Анализ описанной выше модели мы проведем по предложенной в работе [14] схеме. В отличие от рассмотренного в ней случая, в нашей задаче абсолютно непрерывная часть оператора $H_{0}$ есть не все пространство $\mathcal{H}$, а подпространство $\mathcal{H}_{2}$, что приводит к изменению некоторых формул. Там, где изменения несущественны, мы приведем только формулировки нужных нам результатов, а их подробные доказательства опустим.

Лемма 1. Операторная функиия

$$
B_{\delta} \ni \lambda \rightarrow \Gamma_{n}^{ \pm}(\lambda) \in L\left(\mathcal{H}^{+} \rightarrow \mathcal{H}^{+}\right)
$$

аналитична по $\lambda$, ее значения есть вполне непрерывные операторы, и

$$
\Gamma_{n}^{ \pm}(\lambda) \rightarrow \Gamma_{\infty}^{ \pm}(\lambda), \quad n \rightarrow \infty,
$$

равномерно по $\lambda$ в метрике $L\left(\mathcal{H}^{+} \rightarrow \mathcal{H}^{+}\right)$. 
ЛЕмма 2. Точка $\mu=1$ есть полюс первого порядка для функиии

$$
\mu \rightarrow\left(\mu-\Gamma_{\infty}^{ \pm}\left(\lambda_{\infty}\right)\right)^{-1} \in L\left(\mathcal{H}^{+} \rightarrow \mathcal{H}^{+}\right)
$$

Это утверждение следует из леммы 1 работы [14], так как в нашем случае

$$
\left\langle\psi_{\infty}, V_{\infty} \psi_{\infty}\right\rangle=\lambda_{\infty} \neq 0
$$

Как и в [14], из лемм 1 и 2 следует

Лемма 3. Существуют такие числа $\epsilon>0, \delta>0, \quad n_{0}<\infty$, что при $n \geqslant n_{0}$, $\left|\lambda-\lambda_{\infty}\right|<\delta$ проектор

$$
P_{n}^{+}(\lambda)=\frac{1}{2 \pi i} \oint_{|1-\mu|=\epsilon} R\left(\mu, \Gamma_{n}^{+}(\lambda)\right) d \mu
$$

одномерен, аналитически зависит от $\lambda$ и удовлетворяет уравнению

$$
\Gamma_{n}^{+}(\lambda) P_{n}^{+}(\lambda)=P_{n}^{+}(\lambda) \Gamma_{n}^{+}(\lambda)=\mu_{n}^{+}(\lambda) P_{n}^{+}(\lambda)
$$

причем

$$
P_{n}^{+}(\lambda) \rightarrow P_{\infty}^{+}(\lambda), \quad n \rightarrow \infty .
$$

Равенство (15) есть определение числа $\mu_{n}^{+}(\lambda)$.

Лемма 4. Проектор (14) может бъть вычислен по формуле

$$
P_{n}^{+}(\lambda) f=c_{n}(\lambda)\left\langle V_{n} l^{-1}\left(\psi_{n}(\lambda)\right), f\right\rangle \psi_{n}(\lambda),
$$

$2 \partial e$

$$
\begin{aligned}
\mu_{n}^{+}(\lambda) \psi_{n}(\lambda) & =\Gamma_{n}^{+}(\lambda) \psi_{n}(\lambda), \quad \psi_{n}(\lambda) \in \mathcal{H}^{+}, \\
c_{n}(\lambda) & =\left\langle V_{n} l^{-1}\left(\psi_{n}(\lambda)\right), \psi_{n}(\lambda)\right\rangle^{-1}
\end{aligned}
$$

и равномерно по $\lambda$ существуют предельи $\lim _{n \rightarrow \infty} \mu_{n}^{+}(\lambda)=\mu_{\infty}^{+}, \quad \lim _{n \rightarrow \infty} c_{n}(\lambda)=$ $c_{\infty}(\lambda)$ и в метрике $\mathcal{H}^{+} \quad \lim _{n \rightarrow \infty} \psi_{n}(\lambda)=\psi_{\infty}(\lambda)$.

Лемма 5. Справедлива формула

$$
\left.\partial_{\lambda} \mu_{\infty}^{+}(\lambda)\right|_{\lambda=\lambda_{\infty}}=-\frac{1}{\lambda_{\infty}}
$$

Лемма 6. При достаточно больиом $n$ функиия $B_{\delta} \ni \lambda \rightarrow 1-\mu_{n}^{+}(\lambda)$ имеет единственный нуль, и это есть нуль первого порядка:

$$
\mu_{n}^{+}\left(\lambda_{n}\right)=1 \text {. }
$$

Равенство (17) есть определение числа $\lambda_{n}$. Положим

$$
T_{n}^{+}(\lambda)=V_{n}+V_{n} R^{+}\left(\lambda, H_{n}\right) V_{n}, \quad\left|\lambda-\lambda_{\infty}\right|<\delta, \quad \lambda \neq \lambda_{n} .
$$


ЛЕмма 7. При достаточно больиих $n$ оператор (18) может быть записан в виде

$$
T_{n}^{+}(\lambda)=r_{n}(\lambda)+T_{n}^{+}(\lambda)_{\text {reg }},
$$

где оператор $T_{n}^{+}(\lambda)_{\mathrm{reg}}$ аналитичен по $\lambda$ в круге $B_{\delta}$ как функция со значениями в $L\left(\mathcal{H}^{+} \rightarrow \mathcal{H}^{-}\right)$и равномерно по $\lambda$ имеет предел

$$
\left\|\left(T_{n}^{+}(\lambda)_{\text {reg }}-T_{\infty}^{+}(\lambda)_{\text {reg }}\right) \mid L\left(\mathcal{H}^{+} \rightarrow \mathcal{H}^{-}\right)\right\| \rightarrow 0, \quad n \rightarrow \infty
$$

a оператор $r_{n}(\lambda)$ дается формулой

$$
r_{n}(\lambda) f=\frac{c_{n}(\lambda) \mu_{n}^{+}(\lambda)}{1-\mu_{n}^{+}(\lambda)}\left\langle V_{n} l^{-1}\left(\psi_{n}(\lambda)\right), f\right\rangle V_{n} \psi_{n}(\lambda)
$$

При $\operatorname{Im} \lambda>0$ справедлива формула

$$
R\left(\lambda, H_{n}\right)=R\left(\lambda, H_{0}\right)+R\left(\lambda, H_{0}\right) T_{n}^{+}(\lambda) R\left(\lambda, H_{0}\right) .
$$

Из (19) и (20) следует, что точка $\lambda_{n}$ есть полюс аналитического продолжения резольвенты $R\left(\lambda, H_{n}\right)$, и поэтому $\operatorname{Im} \lambda_{n}$ можно оценить на основе “золотого правила Ферми" . В нашем случае удобно воспользоваться либо формулой (9.35) из работы [11], либо формулой (28) из работы [9]. Используя эти формулы и условие (5), мы получаем следуюшее утверждение.

ЛЕмма 8. При $n_{0}<n<\infty$ справедливо неравенство

$$
\operatorname{Im} \lambda_{n}<0
$$

ЛЕмма 9. Если функиия $\psi_{n}(\lambda)$ удовлетворяет уравнению (16), то при

$$
\lambda \in\left(\lambda_{\infty}-\delta, \lambda_{\infty}+\delta\right)
$$

справедливо равенство

$$
\operatorname{Im} \mu_{n}^{+}(\lambda)\left\langle\psi_{n}(\lambda), V_{n} \psi_{n}(\lambda)\right\rangle=-\pi\left\|U\left(P_{2} V_{n}\left(\psi_{n}(\lambda)\right)\right) \mid L^{2}(\Omega, d \omega)\right\|^{2}
$$

Из равенства (21) следует

Лемма 10. Справедливо равенство

$$
U\left(V_{\infty} \psi_{\infty}\right)\left(\lambda_{\infty}, \omega\right) \equiv 0
$$




\section{4. ОСНОВНЫЕ РЕЗУЛЬТАТЫ}

Определим волновые операторы и оператор рассеяния равенствами

$$
\begin{aligned}
W_{ \pm}\left(H_{n}, H_{0}\right) & =\lim _{t \rightarrow \pm \infty} e^{i t H_{n}} e^{-i t H_{0}} P_{2}, \\
S\left(H_{n}, H_{0}\right) & =W_{+}^{\star}\left(H_{n}, H_{0}\right) W_{-}\left(H_{n}, H_{0}\right),
\end{aligned}
$$

где $P_{2}$ - проектор на абсолютно непрерывное подпространство оператора $H_{0}$, т.е. пространство $\mathcal{H}_{2}$. Сушествование и полнота волновых операторов следуют из предположения о ядерности оператора $V_{n}$. При меньших предположениях сушествование и полнота волновых операторов доказаны в работе [2]. Оператор рассеяния коммутирует с $H_{0}$ и в пространстве $h$ задается операторной функцией [15]

$$
\lambda \rightarrow S_{n}(\lambda) \in L\left(L^{2}(\Omega, d \omega) \rightarrow L^{2}(\Omega, d \omega)\right),
$$

которая в окрестности $\lambda_{\infty}$ имеет вид

$$
S_{n}(\lambda)=1-2 \pi i t_{n}^{+}(\lambda), \quad \lambda \in B_{\delta},
$$

где $t_{n}^{+}(\lambda)$ - интегральный оператор в $L^{2}(\Omega, d \omega)$ :

$$
\begin{aligned}
t_{n}^{+}(\lambda) f\left(\lambda, \omega_{1}\right) & =\int t_{n}^{+}\left(\lambda, \omega_{1}, \omega_{2}\right) f\left(\lambda, \omega_{2}\right) d \omega_{2}, \\
t_{n}^{+}\left(\lambda, \omega_{1}, \omega_{2}\right) & =U\left(T_{n}^{+}\left(U^{-1}\left(\lambda, \omega_{2}\right)\right)\left(\lambda, \omega_{1}\right) .\right.
\end{aligned}
$$

Из лемм 7 и 10 следует

ЛЕмма 11. Справедлива формула

$$
\begin{gathered}
t_{n}^{+}\left(\lambda, \omega_{1}, \omega_{2}\right)=\frac{c_{n}(\lambda) \mu_{n}^{+}(\lambda)}{1-\mu_{n}^{+}(\lambda)}\left\langleV _ { n } l ^ { - 1 } \left(\psi_{n}(\lambda), U^{-1}\left(\lambda, \omega_{2}\right\rangle_{2} \times\right.\right. \\
\times U\left(P_{2} V_{n} \psi_{n}(\lambda)\right)\left(\lambda, \omega_{1}\right)+t_{n}^{+}\left(\lambda, \omega_{1}, \omega_{2}\right)_{\mathrm{reg}},
\end{gathered}
$$

$2 \partial e$

$$
t_{n}^{+}\left(\lambda, \omega_{1}, \omega_{2}\right)_{\mathrm{reg}}=U\left(P_{2} T_{n}^{+}(\lambda)_{\mathrm{reg}} U^{-1}\left(\lambda, \omega_{2}\right)\left(\lambda, \omega_{1}\right) .\right.
$$

Лемма 12. 1. Равномерно по $\lambda \in B_{\delta}, \omega_{1}, \omega_{2} \in \Omega$

$$
t_{n}^{+}\left(\lambda, \omega_{1}, \omega_{2}\right)_{\mathrm{reg}} \rightarrow t_{\infty}^{+}\left(\lambda, \omega_{1}, \omega_{2}\right)_{\mathrm{reg}}, \quad n \rightarrow \infty .
$$

2. При фиксированном $\lambda, 0<\left|\lambda-\lambda_{\infty}\right|<\delta$, равномерно по $\omega_{1}, \omega_{2} \in \Omega$

$$
t_{n}^{+}\left(\lambda, \omega_{1}, \omega_{2}\right) \rightarrow t_{\infty}^{+}\left(\lambda, \omega_{1}, \omega_{2}\right), \quad n \rightarrow \infty .
$$

ЛЕмма 13. Если отображение р удовлетворяет условию 9, то

$$
\lim _{\left|\lambda-\lambda_{\infty}\right| \rightarrow+0} \sup _{\omega \in \Omega}\left|t_{\infty}^{+}(\lambda, \omega, p(\omega))_{\text {reg }}-t_{\infty}^{+}\left(\lambda_{\infty}, \omega, p(\omega)\right)\right|=0 .
$$


ДоКАЗАТЕЛЬСТвО. Пусть

$$
W(\lambda, \omega)=U\left(P_{2} V_{\infty} \psi_{\infty}(\lambda)\right)(\lambda, \omega) .
$$

Из леммы 7, уравнений (13) и (19) следует равенство

$$
\left|t_{\infty}^{+}(\lambda, \omega, p(\omega))_{\mathrm{reg}}-t_{\infty}^{+}\left(\lambda_{\infty}, \omega, p(\omega)\right)\right|=\left|c_{\infty}(\lambda) \mu_{\infty}^{+}(\lambda)\left(1-\mu_{\infty}^{+}(\lambda)\right)^{-1}\right||W(\lambda, \omega)|^{2} .
$$

Функция $\lambda \rightarrow W(\lambda, \omega)$ аналитична по $\lambda$ в $B_{\delta}$ как функция со значениями в $C(\Omega)$, и в силу леммы 8 в точке $\lambda_{\infty}$ имеет нуль. Функция $\lambda \rightarrow 1-\mu_{\infty}^{+}(\lambda)$ в точке $\lambda_{\infty}$ имеет нуль первого порядка, что и доказывает лемму.

Из леммы 13 следует

Лемма 14. Справедливо равенство

$$
\lim _{\left|\lambda-\lambda_{\infty}\right| \rightarrow+0} \int\left|t_{\infty}^{+}(\lambda, \omega, p(\omega))_{\mathrm{reg}}-t_{\infty}^{+}\left(\lambda_{\infty}, \omega, p(\omega)\right)\right| d \omega=0 .
$$

Положим

$$
\text { v. p. } t_{\infty}^{+}\left(\lambda_{\infty}, \omega, p(\omega)\right)=\lim _{\left|\lambda-\lambda_{\infty}\right| \rightarrow+0} t_{\infty}^{+}(\lambda, \omega, p(\omega)) .
$$

Основным результатом нашей работы является

Теорема. Если последовательность действительных чисел $\nu_{n}$ удовлетворяет условию

$$
\left|\nu_{n}-\operatorname{Re} \lambda_{n}\right|=O\left(\left|\operatorname{Im} \lambda_{n}\right|^{1+\epsilon}\right), \quad \epsilon>0,
$$

то справедливы равенства

$$
\begin{array}{r}
\lim _{n \rightarrow \infty} \int \mid t_{n}^{+}\left(\nu_{n}, \omega, p(\omega)\right)-\text { v. p. } t_{\infty}^{+}\left(\lambda_{\infty}, \omega, p(\omega)\right) \mid d \omega=\frac{|\beta|}{\pi}, \\
\lim _{n \rightarrow \infty} \int\left|t_{n}^{+}\left(\nu_{n}, \omega, p(\omega)\right)-t_{\infty}^{+}\left(\nu_{n}, \omega, p(\omega)\right)\right| d \omega=\frac{|\beta|}{\pi} .
\end{array}
$$

Доказательство этой теоремы основано на леммах 1-14 и повторяет доказательство теоремы 2 из работы [14], поэтому мы не будем его здесь приводить.

Чтобы в рамках предложенной модели математически корректно рассмотреть упомянутый во введении пример, нужно в качестве исходных операторов взять экспоненты от гамильтонианов $H$ и $H_{0}$. Соответствуюшие рассуждения очень громоздки и лишь в несушественных деталях будут отличаться от приведенных в работе [16], поэтому мы ограничимся формальной схемой.

Положим $\mathcal{H}_{1}=\mathbb{C}^{1}, \mathcal{H}_{2}=L^{2}\left(\mathbb{R}^{1}, d x\right), \mathcal{H}_{2}^{ \pm}=L^{2}\left(\mathbb{R}^{1}, \exp (\mp|x|) d x\right)$. Пусть $v_{n, 11}$ - оператор умножения на действительное число $\lambda_{\infty}>0, v_{n, 22}$ - оператор умножения на функцию $\kappa v_{0}(x)$,

$$
\begin{gathered}
v_{n, 12}: \psi_{2}(x) \rightarrow \epsilon_{n} \int \eta(x) \psi_{2}(x) d x, \\
v_{n, 21}: \psi_{1} \rightarrow \epsilon_{n} \psi_{1} \eta(x), \\
v_{0}(x)=v_{0}(-x) \geqslant 0, \quad \eta(x)=\eta(-x), \quad v_{0}, \eta \in C_{0}^{\infty}\left(\mathbb{R}^{1}\right), \\
\operatorname{supp} v_{0} \cap \operatorname{supp} \eta=\varnothing, \quad \epsilon_{n} \rightarrow 0 .
\end{gathered}
$$


Положим $\Omega=\{-1,1\}$ и припишем каждой точке \pm 1 меру 1 . Пусть

$$
\begin{gathered}
\hat{f}(k)=\int e^{-i k x} f(x) d x, \quad h=L^{2}(\Omega \times[0, \infty), d \omega d \lambda) \\
U f(\lambda, \omega)=(4 \pi \sqrt{\lambda})^{-1 / 2} \hat{f}(\omega \sqrt{\lambda}), \quad U^{-1}(x \mid \lambda, \omega)=(4 \pi \sqrt{\lambda})^{-1 / 2} e^{i x \omega \sqrt{\lambda}} .
\end{gathered}
$$

Отображения $l$ и $p$ можно определить двумя способами: можно положить $l \psi(x)=\bar{\psi}(x)$, $p(\omega)=-\omega$ или $l \psi(x)=\bar{\psi}(-x), p(\omega)=\omega$.

Выполнение всех условий (конечно, за исключением ядерности и вполне непрерывности) проверяется тривиально, и $|\beta|=1$. Величина $1-2 \pi i t_{n}^{+}(\lambda, \omega, \omega)$ - коэффициент прохождения плоской волны, идушей из $-\infty$ в $+\infty$ из $+\infty$ в $-\infty), \mathrm{a}-2 \pi i t_{n}^{+}(\lambda,-\omega, \omega)-$ коэффициент отражения. Отсюда следуют сделанные во введении утверждения.

Благодарности. Работа вьполнена при поддержке гранта РФФИ № 02-01-00271.

\section{Список литературы}

[1] Б. О. Кербиков. ТМФ. 1985. Т. 65. № 3. С. 379-390.

[2] R.F. Dashen, J. B. Healy, I. J. Muzinich. Ann. Phys. 1976. V. 102. P. 1-70.

[3] R.F. Dashen, J. B. Healy, I. J. Muzinich. Phys. Rev. D. 1976. V. 14. P. 2773-2789.

[4] J. Dereziński, V. Jukšić. J. Funct. Anal. 2001. V. 180. P. 243-327.

[5] K. Фридрихс. Возмущение спектра операторов в гильбертовом пространстве. М.: Мир, 1969.

[6] J. S. Howland. J. Math. Anal. Appl. 1975. V. 50. P. 415-437.

[7] G. Parravicini, V. Gorini. J. Math. Phys. 1980. V. 21. P. 2208-2226.

[8] Ж.И. Абдулаев, И. А. Икромов, С.Н. Лакаев. ТМФ. 1995. Т. 103. С. 54-62; А.К. Мотовилов. ТМФ. 1996. Т. 107. С. 450-477; С. 478-500; Р. Менникен, А. К. Мотовилов. ТМФ. 1998. Т. 116. С. 163-181; В. Б. Беляев, А.К. Мотовилов. ТМФ. 1997. Т. 111. С. 77-93; Ю. А. Куперин, К. А. Макаров, С. П. Меркурьев, А. К. Мотовилов, Б. С. Павлов. ТМФ. 1988. Т. 75. С. 431-444; Т. 76. С. 242-260.

[9] Ю.А. Куперин, К.А. Макаров, С.П. Меркурьев, А.К. Мотовилов. ЯФ. 1988. Т. 48. C. $358-370$.

[10] R. Mennicken, A.K. Motovilov. Math. Nachr. 1999. B. 20. S. 117-181; A. K. Motovilov. J. Math. Phys. 1991. V. 32. P. 3509-3518; A.K. Motovilov, W. Sandhas, V. B. Belyaev. J. Math. Phys. 2001. V. 42. P. 2490-2506.

[11] S. Agmon. Commun. Pure Appl. Math. 1998. V. 51. P. 1255-1309.

[12] S. Albeverio, R. Hoegh-Krohn. J. Math. Anal. Appl. 1984. V. 101. P. 491-513.

[13] К. Морен. Методы гильбертова пространства. М.: Мир, 1965.

[14] А. А. Арсеньев. ТМФ. 2003. Т. 134. С. 341-352.

[15] Д. Р. Яфаев. Математическая теория рассеяния. Санкт-Петербург: Изд-во Санкт-Петербургского университета, 1994.

[16] А. А. Арсеньев. Матем. сб. 1996. Т. 187. С. 3-20. 